\title{
Enteroparasitosis infantil en el Area Metropolitana de Salud Central de Santiago
}

Dres. Carlos Pérez B. y Morelia Vallejos P."*

Los informes estadísticos de las enteroparasitosis en Chile se inician en 1956, año en que Neghme y Silva ${ }^{1}$ publican los resultados de las primeras encuestas. Posteriormente diversos autores realizan nuevos estudios en el país $^{2 \cdot 3 \cdot 4-6-7-8-9-10}$.

En el presente trabajo se analiza la frecuencia de la enteroparasitosis diagnosticada en el Laboratorio de Parasitología del Hospital de Niños "Manuel Arriarán" de Santiago de Chile, que sirve a ese hospital y a la población infantil de los consultorios periféricos Caimi, Maipú, Cerrillos, Lo Valledor Norte, Chuchunco y Nogales, que pertenecen al Area Metropolitana de Salud Central de Santiago, que atiende a 143.946 menores de 14 años.

\section{MATERIAL Y METODO}

Se analizan estadísticamente los exámenes coproparasitarios y test de Graham efectuados desde el año 1972 a 1975. Las tres muestras de deposiciones entregadas por cada paciente fueron sometidas al método de Telemann modificado ${ }^{11}$ y la prueba de Graham se hizo examinando cinco placas. En total se recopilaron 13.203 exámenes coproparasitarios y 2.415 test de Graham.

\footnotetext{
* Departamento de Medicina Experimental, Unidad de Parasitologia, Facultad de Medicina Sur Universidad de Chile, Santiago de Chile.

* Iaboratorio de Gastroenterología, Hospital Manuel Arriarin, Santiago de Chile.
}

\section{RESLLTADOS}

En las tablas 1 y 2 presentamos por grupos de edades la frecuencia de los diversos protozoos $\mathrm{y}$ helmintos intestinales diagnosticados.

De los 13.203 exámenes de deposiciones efectuadas, se observaron elementos parasitarios o comensales en $6.389(48,4 \%)$ y solamente parásitos en $4.855(36,8 \%)$.

Entre los protozoos, Giardia lamblia es el parásito más frecuente $(29,9 \%)$; la tasa más alta se encontró en el grupo de niños de 1 a 4 años, llegando al $37,1 \%$. Le siguen en porcentaje los protozoos comensales Entamoeba coli $(19,4 \%)$ y Endolimax nana (16,6\%). Entamoeba histolytica se encuentra en baja frecuencia $(3,3 \%)$, siendo los menores de un año los menos parasitados; su incidencia se eleva a mayor edad, llegando a $7,7 \%$ entre 10 y 14 años.

El helminto más frecuentemente detectado fue Hymenolepis nana $(3,1 \%)$; le siguen Ascarts lumbricoides $(2,1 \%)$ y Trichuris trichiura con $1,8 \%$.

De los 2.415 test de Graham examinados 788 estaban positivos $(32,6 \%)$.

\section{DISCUSION}

Las infecciones por protozoos intestinales son más frecuentes que las por helmintos en los exámenes coproparasitarios $y$ concuerda con lo informado por otros autores ${ }^{1-2 \cdot 7-10}$.

Las cifras de infección por $E$. histolytica y G. lamblia encontradas por nosotros son similares a las de Rubio ${ }^{2}$ y Noemí y col. ${ }^{10}$, siendo 
más bajas que las publicadas por Neghme $y$ Silva' y Ramírez y col.?

Llama la atención que en los menores de un año no se encontraron, fuera de $G$. lamblia $(7,9 \%)$, otras infecciones parasitarias, lo que indicaría que la patología digestiva presentada por estos niños y que sería el motivo de solicitar examen parasitológico de deposiciones, obedecería a causas no parasitarias.

En nuestro estudio las infecciones encontradas por metazoos intestinales son más bajas que las publicadas por Noemi y col. ${ }^{10}$. Hymenolepis nana, con un $3,1 \%$, es el helminto con más alta tasa. A. lumbricoides con $2,1 \%$ y $T$. trichiura con $1,8 \%$ presentaron los valores más bajos en este grupo de parásitos. En nuestra investigación igual que en la de Rubio ${ }^{2}$ y Noemi y col. $^{10}$ constatamos que $A$. lumbricoides y $T$. trichiura se presentaron en menor porcentaje que $H$. nana discordando con los demás autores ${ }^{5.7912}$ que encuentran una mayor incidencia de $A$. lumbricoides y $T$. trichiura.

Desde el año 1963, Neghme y Silva ${ }^{3}$ sospechan una tendencia decreciente de infecciones por $A$. lumbricoides y $T$. trichiura, hecho que se confirma con nuestro trabajo y el de Noemí y col. ${ }^{10}$, una situación similar está ocurriendo con $H$. nana.

Otras teniasis prácticamente no se han diagnosticado, sólo en 11 de los 13.203 exámenes presentaron huevos de Taenia sp. $(0,08 \%)$.

Huevos de Oxyuris vermiculares en deposiciones fue un hallazgo en 90 casos $(0,7 \%)$, cifra que es muy inferior al 1,6 y $8 \%$ encontrada por Torres y col. ${ }^{9}$ y Biefang ${ }^{13}$ en un grupo de Valdivia y La Unión, respectivamente.

Los valores de positividad detectados en el test de Graham $(32,6 \%$ ) son bajos por tratarse de pacientes seleccionados, a los cuales en general se solicita este examen por presentar alguna sospecha clínica de esta parasitosis. Neghme $y$ col. $^{12}$, en una encuesta de 1.029 escolares de Santiago encuentran un $36,48 \%$ de positividad. En alumnos de internados se han encontrado cifras de $87,8 \%{ }^{6}$ y $90,1 \%{ }^{14}$.

\section{RESUMEN}

Se estudia la incidencia de enteroparasitosis en 13.203 exámenes coproparasitarios solicitados al Hospital Manuel Arriarán. de Santiago de Chile, en el período comprendido entre 1972 y 1975

Se encontraron parásitos y/o comensales en 6.389 exámenes $(48,4 \%)$, sólo parásitos en 4.855 exámenes $(36,8 \%)$ y negativos $6.814(51,6 \%)$.

Giardia lamblia es el protozoo parásito de mayor frecuencia con un $29,9 \%$, con su máxima incidencia entre 1 y 4 años $(37,1 \%)$; Entamoeba histolvitica se encontró en un $3,3 \%$, con mayor incidencia entre los 10 y 14 años $(7,7 \%)$.

Éntre los helmintos se constató Hymenoleprs nana, $3,1 \%$; Ascaris lumbncoides, $2,1 \%$ y Tnchuris trichiura, $1,8 \%$.

Durante este periodo, se efectuaron 2.415 test de Graham resultando positivos $788(32,6 \%)$.

\section{SUMMARY}

The incidence of enteric parasites in 13.203 stools for ova and parasites, performed at the Manuel Arriaran Pediatric Hospital, Santiago, Chile, from 1972 to 1975 is studied.

Parasites and/or commensals are found in 6,389 samples $(48,4 \%)$, parasites in 4,855 samples $(36,8 \%)$ and normal specimen in $6,814(51,6 \%)$.

Out of 13,203 stool specimen examined for ova and parasite, Ciardia lamblia is the most frequent $(29,9 \%)$. The highest incidence was between 1 and 4 years of age $(37,1 \%)$. Entamoeba histolyttca was found in $3,3 \%$ with its highest incidence between 10 and 14 years of age $(7,7 \%)$.

Among helminths: Hymenolepis nana $3.1 \%$, Ascaris lumbncotres $21 \%$, and Trichuris truhuira $1.8 \%$ were found.

During this period, 2,415 Graham tests were done. $32.6 \%$ were positive.

Tabla 1

INFECCIONES POR PROTOZOOS INTESTINALES DIAGNOSTICADOS EN 13.203 EXAMENES COPROPARASITARIOS EN EL HOSPITAL MANUEL ARRIARAN, SANTIAGO-CHILE, 1972-1975

\begin{tabular}{|c|c|c|c|c|c|c|c|c|c|c|c|}
\hline \multirow{2}{*}{ Edad años } & \multirow{2}{*}{ Exámenes } & \multirow{2}{*}{$\begin{array}{c}\text { Crabrtia } \\
N^{\circ}\end{array}$} & \multirow{2}{*}{$\begin{array}{c}\text { lam- } \\
\text { blia } \\
\%\end{array}$} & \multicolumn{2}{|c|}{ Entamoebahtstolytica } & \multicolumn{2}{|c|}{ Entamoebacoli } & \multicolumn{2}{|c|}{ Endolimax nana } & \multicolumn{2}{|c|}{ Chilomastix mesnil } \\
\hline & & & & $N^{\circ}$ & $\%$ & $N^{\circ}$ & $\%$ & $\mathrm{~N}^{*}$ & $\%$ & $N^{\circ}$ & $\%$ \\
\hline 0 a 1 & 1.920 & 152 & 7,9 & 6 & 0,3 & 49 & 2,6 & 24 & 1,3 & 6 & 0,3 \\
\hline 1 a 4 & 2.885 & $1.0^{7} 1$ & 37,1 & 45 & 1,6 & 437 & 15,1 & 297 & 10,3 & 64 & 2,9 \\
\hline 5 a 9 & 2.106 & 733 & 34,8 & 106 & 5,0 & 585 & 27,8 & 528 & 25,1 & 50 & 2,4 \\
\hline 10 a 14 & 1.136 & 285 & 25,1 & 87 & 7,7 & 413 & 36,4 & 385 & 33.9 & 41 & 3,6 \\
\hline Edad desconocida & a 5.156 & 1.704 & 33,0 & 192 & 3.7 & 1.078 & 20,9 & 957 & 18,6 & 143 & 2,8 \\
\hline Total & 13.203 & 3.945 & 29,9 & 436 & 3,3 & 2.562 & 19,4 & 2.191 & 16,6 & 304 & 2,3 \\
\hline
\end{tabular}


Tabla 2

INFECGIONES POR HELMINTOS INTESTINALES DIAGNOSTICADOS EN

13.203 EXAMENES COPROPARASITARIOS EN EL HOSPITAL MANUEL ARRIARAN,

SANTIAGO CHILE. 1972-1975

\begin{tabular}{|c|c|c|c|c|c|c|c|}
\hline \multirow[t]{2}{*}{ Edad años } & \multirow[t]{2}{*}{ Exámenes } & \multicolumn{2}{|c|}{ Hymenolepis nana } & \multicolumn{2}{|c|}{ Ascaras lumbricoides } & \multicolumn{2}{|c|}{ Trichure trichura } \\
\hline & & $\mathrm{N}^{\circ}$ & $\%$ & & $\%$ & $\mathrm{~N}^{\circ}$ & $\%$ \\
\hline 0 a 1 & 1.920 & 5 & 0,2 & - & - & 2 & 0,1 \\
\hline 1 a 4 & 2.885 & 91 & 3.2 & 69 & 2,4 & 56 & 1,9 \\
\hline 5 a 9 & 2.106 & 103 & 4,9 & 51 & 2,4 & 62 & 2,9 \\
\hline 10 a 14 & 1.136 & 55 & 4,8 & 23 & 2,0 & 31 & 2,7 \\
\hline Edad desconocida & 5.156 & 158 & 3,1 & 128 & 2,5 & 85 & 1,6 \\
\hline Total & 13.203 & 412 & 3,1 & 271 & 2,1 & 236 & 1,8 \\
\hline
\end{tabular}

\section{REFERENCIAS}

1 Veghme, $A$ y Silva, $R$. Distribución y frecuencia de las enteroparasitosis en Chile. Rev. Serv. Nac. Salud, Chile 1:3-24, 1956.

2 Rubio, M. Encuesta enteroparasitológica en el Hospital de Niños "Luis Calvo Mackenna" en Santiago. Consideraciones clínicas y epidemiológicas sobre 270 casos. Bol. Chile. Parasit. 17: 93-99, 1962.

3 Neghme, A. y Silva, $R$. Estado actual de las infecciones por Ascans lumbricoides y Trichuris trichiura en Chile. Distribución geográfica y prevalencia. Bol. Chile. Parasit. 18:100-103, 1963.

- Bull, F.; Oyarce, R. y Stehr, I. Contribución al estudio y prevalencia de parasitosis intestinales en algunas poblaciones de la provincia de Concepción (con especial referencia a Entamoeba histolytica y otros protozoos intestinales). Bol. Chile. Parasit. 19:110-114, 1964.

- Bull, E.; Oyarce, R. y Stehr, l. Contribución al estudio de prevalencia de parasitosis intestinales en algunas poblaciones de la provincia de Concepción (con especial referencia a helmintos $\rangle$. Bol. Chile. Parasit. 22: 10-15, 1967 .

- Cuevas, R.; Schenone, H.; Silva, R.; Galdames, M.; Inzunza, E. y Romero, $E$. Prevalencia de infecciones por Enterobius vermicularis en un internado. Bol. Chile. Parasit. 24:121-123, 1969.
7 Ramírez, R.: Schenone, H.; Galdames, M.; Romero, E.; Inzunza, E.; Rojas, A.; Palomino, H. y Szekely, $R$. Frecuencia en Chile de las infecciones humanas por protozoos y helmintos intestinales (1962-1972). Bol. Chile. Parasit. 27:116-118, 1972.

s Puga, S. Protozoos y helmintos intestinales en lactantes de la ciudad de Valdivia, Chile, Bol. Chile. Parasit. 28:116-118, 1972.

- Torres, P.; Figueroa, L.; Puga, S.; Franjola, R.; Navarrete, N.; Momberg, J.; Valdivia, L.; Filipich, L.; Mohr, P.; Cabezas, P. y Barría, $H$. Protozoos y helmintos intestinales en la población escolar de la ciudad de Valdivia, Chile. Bol. Chile. Parasit. 29:112-114, 1974.

10 Noemi, 1.; Reyes; H. y Mowro, G. Enteroparasitosis en la población infantil del Area de Salud Oriente de Santiago. Rev. Chile. Pediatr. 45:454-457, 1974.

11 Doien, G.; Galdames, M. y Silva, R. Algunas consideraciones sobre el rendimiento de las técnicas de diagnóstico de enteroparasitosis. Bol. Chile. Parasit. 1.3: 42-44, 1958

12 Neghme, A.; Silva, $R$. y Sotomayor, $R$. Enteroparasitosis entre escolares de la provincia de Santiago. Bol. Chile. Parasit. 9:70-79, 1954.

13 Brefang, F. y López, J. Estudio parasitológico de deposiciones en alumnos de una escuela urbana de La Unión. Bol. Chile. Parasit. 25:142-143, 1970.

14 Schenone, H.; Arias, B.; Galdames, M.; Subzabre, V.; Cuevas, R.; Inzunza, E.; Romero, E. y Jiménez. M. Rendimiento de los exámenes seriados en el diagnóstico de laboratorio de la infección por Enterobios vermicularis. Bol. Chile. Parasit. 25:113-117, 1970. 